\title{
circ-ANKS1B facilitates osteosarcoma cell proliferation and invasiveness via upregulating Ki- 67 expression by sponging miR-149-5p
}

Hao Yang

Zhengzhou University

Yujie Liu

Zhengzhou University

Chao Li

Zhengzhou University

Yilin Liu

Zhengzhou University

Liang Zhao

Zhengzhou University

Yuqiang Wang

Zhengzhou University

Yang Zhang

Zhengzhou University

Min Zhang

Zhengzhou University

Limin Wang ( $\square$ zzuwanglimin@163.com )

Zhengzhou University https://orcid.org/0000-0002-6344-0738

\section{Primary research}

Keywords: circ-ANKS1B, miR-149, progression, osteosarcoma, Ki-67

Posted Date: February 13th, 2020

DOI: https://doi.org/10.21203/rs.2.22619/v2

License: (c) (i) This work is licensed under a Creative Commons Attribution 4.0 International License.

Read Full License 


\section{Abstract}

Background: Mounting evidence has shown that Circular RNAs (circRNAs) are associated with initiation and progression of human cancers. However, the expression and function of circRNAs in the development of osteosarcoma (OS) remain unclear.

Methods: In this study, the expression profiles of circRNA circ-ANKS1B in OS were identified through qRTPCR and in situ hybridization (ISH). The relationships between expression of circ-ANKS1B and clinicopathological features of OS patients was analyzed. Cell proliferation potential, migration and invasion ability of OS cells were evaluated through CCK8, colony formation, transwell and wound healing assays in vitro. Xenograft nude mouse experiment was performed to investigate tumor formation ability in vivo. The downstream regulated microRNA of circ-ANKS1B was proved via qRT-PCR and dualluciferase reporter.

Results: We found expression of circ-ANKS1B was markedly overexpressed in OS cell lines and tumor tissues, and high expression of circ-ANKS1B was correlated with advanced TNM stage and poor prognosis of OS patients. The results of functional experiments showed that depletion of circ-ANKS1B could inhibit proliferation and invasion ability of OS cells in vitro, and tumor formation ability in vivo. Further mechanistic studies revealed that circ-ANKS1B could sponge endogenous miR-149-5p and partially reversed the suppressive effect of miR-149-5p in OS cells. Furthermore, we demonstrated that circ-ANKS1B regulated Ki-67 expression by sponging miR-149-5p. Conclusions: In summary, our data showed that circ-ANKS1B accelerated cell growth and invasion in OS by sponging miR-149-5p and regulating Ki-67.

\section{Background}

Osteosarcoma (OS) is one of the most common primary skeletal highly aggressive tumors in children and adolescents[1]. OS frequently originates from transformed cells characterized by immature bone formation or malignant osteoid producing[2]. Although surgical resection combined with neoadjuvant chemotherapy had greatly improved the prognosis of OS, patients with recurrence and metastasis remain suffer a dismal prognosis and the most clinical challenging burdens [3]. It is, hence, urgently needed to identify the molecular mechanisms underpinning the malignant transformation of this deadly disease.

Circular RNAs (circRNAs) are proved to be a special type of non-coding RNAs [4, 5]. Although increasing number of novel circRNAs have been identified, but their biological functions remain largely unknown. Promising evidences have proven that circRNA participates in various physiological and pathological processes[6]. Additionally, several circRNAs have been reported to be closely associated with tumor progression $[7,8]$. circRNAs could indirectly regulate gene expression and cancer progression through the competitive endogenous RNAs (ceRNAs) manner [7]. For instance, circRNA_000284 promotes cervical cancer cell aggressive behaviors by sponging miR-506[9]. In colorectal cancer, circ-CBL. 11 suppresses 
cancer growth through sponging miR-6678-5p[10]. However, the interaction mechanisms of circRNAs and miRNA are waiting for be further explored in OS.

Previous studies also confirmed that multiple circRNAs contribute to the OS progression. Previously, Zhou et al. reported that circRNA-0008717 promoted OS cell proliferation, migration and invasion ability via specific sponging with miR-203[11]. Zhang et al. proved that circRNA UBAP2 could promote OS progression via inhibiting miR-143 through sponge adsorption[12]. Huang et al. showed that circRNA0002052 inhibited OS cell proliferation, migration and invasion through affecting activation of miR1205/APC2 axis[13]. Moreover, it has been suggested that circNASP and circRNA-100876 were involved in the progression of OS $[14,15]$. Therefore, it is essential to reveal the functional role of circRNAs in OS.

In this study, we observed that expression of circ-ANKS1B was elevated in OS tissues and cell lines. Overexpression of circ-ANKS1B expression was correlated with the dismal prognosis of OS patients. Additionally, suppression of circ-ANKS1B could inhibit proliferation, migration and invasion ability in vitro, tumor formation ability in vivo. Moreover, circ-ANKS1B could suppress the Ki-67 level of OS cells via sponging with miR-149. Our findings revealed the crucial role of the circ-ANKS1B/miR-149/Ki-67 axis in OS progression and provide a potential novel therapeutic target for treatment of OS.

\section{Materials And Methods}

\section{Human specimens}

The human study was approved by the Institutional Ethical Board of the First Affiliated Hospital of Zhengzhou University, Zhengzhou Central Affiliated Hospital to Zhengzhou University, the Affiliated Cancer Hospital of Zhengzhou University. All adult patients or parents/legally authorized representatives of participants under age 18 signed the written informed consents before enrolled into the study. All of recruited patients were diagnosed between 2008-2013 were totally enrolled. Surgical removed samples included adjacent benign tissue and primary tumor were fixed in $10 \%$ formalin then embedded with paraffin for immunohistochemistry analyses.

\section{Construction of tissue microarray (TMA)}

We combined all OS samples from the above mentioned three hospital to create a relative large-scale cohort with more OS samples[16, 17]. The osteosarcoma TMA used in present study was constructed with 120 osteosarcoma specimens and 65 normal specimens.

\section{Cell lines and cell culture}

MG63, U2OS, and 143B were purchased from the American Type Culture Collection (Rockville, USA). Normal osteoblast cells $\mathrm{HOBC}$ and HFOB was purchased from Shanghai Institute for Biological Science (Shanghai, China). All cell lines were maintained at $37^{\circ} \mathrm{C}$ in a humidified incubator (Thermo Fisher Scientific, USA) containing $5 \% \mathrm{CO} 2$. 


\section{Immunohistochemical (IHC) and in situ hybridization (ISH)}

IHC was performed as previous description[18]. ISH was performed through digoxygenin (DIG)-labeled circ-ANKS1B ISH probes as described previously[19]. circ-ANKS1B expression in OS tumor tissues and adjacent non-tumor tissues was detected by fluorescence. The intensities and proportion of circ-ANKS1B dyeing was calculated as following method: 1-3 scores were the low expression, while 3-5 scores were represented high expression.

\section{Western blotting}

Western blotting was performed as previous description [20]. And the primary antibodies were as following: Ki-67 (Proteintech, 27309-1-AP), GAPDH (Proteintech, 10494-1-AP).

\section{Quantitative real- time PCR}

Total RNA was obtained via the method of Trizol (Life Technologies, USA) extraction. And the target cDNA was synthesized through the PrimeScript RT reagent Kit (Promega, USA). Quantitative real-time PCR reactions were performed using SYBR Green kit (ABI, USA). The relative gene expression was normalized to control using $2-{ }^{\Delta \Delta} \mathrm{Ct}$ method. The primers used in this study were listed in Supplemental Table 1.

\section{Cell Transfection}

MG63 or U2OS cells $\left(1 \times 10^{6}\right.$ cells/well) were seeded into a 6-well plate. $24 \mathrm{~h}$ later, cells were transiently transfected with the miR-149 mimics or negative control (NC), shRNA targeting circ-ANKS1B or negative control through lipofectamine 3000 (Invitrogen, USA). The RNAi sequences used in this study were listed in Supplemental Table 2.

\section{CCK-8, Cell invasion and Colony formation assay}

CCK-8, cell migration and invasion assays were performed according to previously described method[21].

\section{Dual luciferase reporter gene assay}

PmirGLO, pmirGLO-circ-ANKS1B or pmirGLO-circ-ANKS1B-mut vectors, pmirGLO-Ki-67 or pmirGLO-Ki-67mut vectors were cotransfected with miR-149 by Lipofectamine ${ }^{\mathrm{TM}} 2000$ Transfection Reagent (Invitrogen); 24 hours later, the luciferase intensity was measured and normalized to the renilla luciferase intensity using the Dual-Luciferase ${ }^{\circledR}$ Reporter Assay System (Promega).

\section{Xenograft model}

Male nude mice were ready for subcutaneously injection. U20S cells stably transfected with sh-circANKS1B or ctrl $\left(5 \times 10^{6}\right.$ cells) were inoculated subcutaneously into the flanks of the nude mice. Tumor size and volumes were measured every week. 4 weeks later, all the mice were sacrificed and the tumor 
weights were measured. All animal experiments were approved by the Animal Care Committee of the First Affiliated Hospital of Zhengzhou University. All experimental procedures involving animals were strictly followed in accordance with the Guide for the Care and Use of Laboratory Animals as described previously[20].

\section{Statistical analysis}

All of the statistical analyses were performed using GraphPad Prism software. Two-tailed Student's ttests were used to compare mean values of two groups. Two-way ANOVA was conducted to compare mean values of more than two groups. Pearson $\chi 2$ tests were adopted to analyze the association of circANKS1B expression with clinicopathological variables. For the survival analysis, Kaplan-Meier plots and log-rank tests were used. Two-tailed Fisher's exact test was performed as appropriate. $p$ value $<0.05$ was considered as statistically significant difference.

\section{Results}

circ-ANKS1B is overexpressed in OS tissues and cell lines.

Based on human reference genome GRCh37/hg19, circ_0007294 is located at chr12:100166699100175875 and is assumed to derive from the gene ANKS1B. Therefore, we termed hsa_circ_0007294 as "circ-ANKS1B". To detect the expression of circ-ANKS1B in OS, qRT-PCR was conducted in 30 OS tissues and paired adjacent non-tumor tissues. We observed that circ-ANKS1B was significantly overexpressed in OS tissues compared to matched nonneoplastic counterparts (Fig. 1A, 1B). Similarly, circ-ANKS1B level was also higher in OS cell lines, including MG63, U2OS and 143B cells than that in normal human osteoblast cell lines (HFOB and HOBC) (Fig. 1C). Subsequently, we verified circ-ANKS1B expression in OS TMA using the ISH assay. Based on the result of ISH staining, we divided circ-ANKS1B expression levels into five stratifications (Fig. 1D), then we found that circ-ANKS1B expression was overexpressed in OS tissues comparing with adjacent non-tumor tissues (Fig. 1E). Taken together, we proved that circ-ANKS1B expression is upregulated during OS tumorigenesis.

\section{High expression of circ-ANKS1B predicts poor prognosis of OS patients}

Then, we investigated the correlation between circ-ANKS1B expression and clinicopathological characteristics (Table 1). We found that circ-ANKS1B expression is significantly related to later TNM stage and large tumor size (Fig. 2A, 2B). Meanwhile, circ-ANKS1B expression was higher in metastatic tumors than in primary osteosarcoma tissues (Fig. 2C). Additionally, there was a remarkable tendency for increased circ-ANKS1B expression in recurrent OS tissues (Fig. 2D). Moreover, we found that OS patients who with overexpression of circ-ANKS1B always lead to poor overall survival and disease-free survival (Fig. 2E, 2F). Furthermore, circ-ANKS1B expression was found to be independent prognostic factors by multivariate analysis (Table 2). These findings indicated that circ-ANKS1B might contribute to OS progression and spired us to investigate the biological mechanism of circ-ANKS1B. 


\section{Knockdown of circ-ANKS1B suppresses OS proliferation and invasion in vitro}

To analyze the potential function of circ-ANKS1B, we performed functional experiments. Because circANKS1B level was the highest in U2OS and 143B cells, we knocked down the expression of circ-ANKS1B in these two cell lines using specific shRNAs targeting circ-ANKS1B (Fig. 3A, 3B). Notably, circ-ANKS1B silencing did not affect the expression of its linear mRNA ANKS1B (Fig. 3C). Subsequently, to evaluate the function of circRNA-000284 on cell proliferation, CCK-8 assay was conducted and the results showed that suppression of circ-ANKS1B could dramatically inhibit the cell proliferation in contrast to control cells (Fig. 3D). And we also observed that knockdown of circ-ANKS1B could suppress the colony formation and DNA synthesis rate compared with that in the negative control (Fig. 3E, 3F). Additionally, results of migration and invasion assays suggested that the invasive and migratory capacity was significantly inhibited by transfection of sh-circ-ANKS1B (Fig. 3G, 3H). In a word, these findings indicated that circANKS1B acted as a important role during progression of OS cells.

\section{Depletion of circ-ANKS1B suppresses OS growth in vivo}

To further determine whether circ-ANKS1B could affect OS tumorigenesis in vivo, we conducted stable circ-ANKS1B-depleted U2OS cells and injected them subcutaneously into the flanks of nude mice (Fig. 4A). The circ-ANKS1B-depleted group (sh-circ-ANKS1B) mice developed weaker luciferase signal compared with the control group (NC) (Fig. 4B). At five weeks after inoculation, tumor volume and weight were noticeably decreased in the circ-ANKS1B silencing group compared with the NC group (Fig. 4C and 4D). Moreover, Ki-67 IHC staining intensive from the xenograft tumors also declined in the circ-ANKS1Bdepleted group (Fig. 4E), suggesting that suppression of circ-ANKS1B could inhibit progression of OS in vivo.

\section{circ-ANKS1B functions as a sponge for miR-149-5p}

Given that it has been widely identified that circRNAs exerted biological function mainly through sponging to specific miRNAs, then by using Circinteractome tool (https://circinteractome.nia.nih.gov/), we identified that miR-149-5p, a known tumor suppressor in OS, contains complementary sequence to circ-ANKS1B (Fig. 5A). In addition, Pearson correlation analysis suggested that circ-ANKS1B was negatively correlated with miR-149-5p expression (Fig. 5B). Additionally, we observed that miR-149-5p was evidently low-expressed in OS tissues and cell lines (Fig. 5C, 5D). Moreover, as shown in Fig. 5E and $\mathbf{5 F}$, overexpression circ-ANKS1B significantly inhibited miR-149-5p expression, while circ-ANKS1Bdepleted dramatically increased miR-149-5p expression level. More importantly, luciferase reporter assay was performed, and results confirmed miR-149-5p could directly interact with circ-ANKS1B (Fig. 5G). Taken together, these findings suggest that circ-ANKS1B directly bind to miR-149-5p and inhibit its activity.

circ-ANKS1B affects proliferation and invasion abilities of OS cells via miR-149-5p 
We further examined whether circ-ANKS1B functions as an oncogene by sponging with miR-149-5p, U2OS or 143B cells were transfected with miR-149-5p mimics or mock control, with/without circ-ANKS1B overexpression vector. Cell proliferation and colony formation assays indicated that overexpression of miR-149-5p could inhibit cell proliferation, while overexpression of circ-ANKS1B partially reversed the suppressive effect of miR-149p-5p in U2OS or 143B cells (Fig. 6A and 6B). Consistently, transwell assay showed that circ-ANKS1B reversed the inhibition effect of miR-149-5p on cell invasion ability (Fig. 6C). Based on these results, circ-ANKS1B affected the proliferation and invasion abilities of OS cells, at least partly by miR-149-5p.

\section{circ-ANKS1B-mediated miR-149 negatively regulates Ki-67 in OS}

Recently studies revealed that circRNA could exert biological function by involving in circRNA-miRNAmRNA crosstalk. Based on this, we performed TargetScan to identify the potential gene targeting by miR149-5p. We found the 3'UTR of Ki-67, a well-known tumor proliferation marker, bears one potential miR149-5p binding sites (Fig. 7A). In addition, we found expression of Ki-67 was negative correlated with miR-149-5p in OS samples (Fig. 7B). Luciferase reporter assay was performed to prove the effect of miR149-5p on Ki-67 mRNA level. And the results showed that miR-149-5p could obviously inhibit luciferase activity of Ki-67-3'UTR wild-type compared with negative control (Fig. 7C). Furthermore, we proved that miR-149-5p could significantly regulate the mRNA and protein levels of Ki-67 in OS cells (Fig. 7D and 7E). Co-transfected with circ-ANKS1B and miR-149 mimic could reverse the function of circ-ANKS1B on Ki-67 expression (Fig. 7E). Consistently, Ki-67 was positively correlated with expression of circ-ANKS1B in OS tissues (Fig. 7F).

Functional experiments showed that Ki-67 overexpression reversed the inhibition effect of circ-ANKS1B on cell proliferation (Fig. 8A, 8B), as well as invasion ability (Fig. 8C). These finding indicated that circANKS1B sponges miR-149-5p, which subsequently allows for Ki-67 translation. circ-ANKS1B promotes the proliferation of OS partly via the circ-ANKS1B/miR-149/Ki-67 axis.

\section{Discussion}

Comparing with miRNA and IncRNA, circRNA displayed greater advantages as a biomarker for tumor diagnosis due to highly conserved and stable feature[7]. Increasing evidence have revealed that abnormal expression of circRNAs could significantly affect the progression of tumors through specific sponging with miRNA[8]. Recent studies proved that circRNA_0008035 was markedly overexpressed in gastric cancer tissue, and suppression of circRNA_00080350 could substantially affect the proliferative rate of cells by regulating miR-375/YBX1[22]. Other studies also proved that knockdown of circ_0002024 in bladder cancer could significantly suppresses cell proliferation and invasion by sponging miR-197-3p[23]. circRNA_0103809 promoted progression of hepatocellular carcinoma through regulating activation of miR-490-5p/SOX2 signaling pathway[24]. All these findings demonstrated that circRNAs acted as important role during initiation and progression of tumors. 
In this study, we uncovered that circRNA circ-ANKS1B was up-regulated in the OS tissues and cell lines for the first time. We found that high level of circ-ANKS1B was significantly associated with advanced TNM stage, highly metastasis and recurrence incidence. Additionally, high expression of circ-ANKS1B always lead to poor prognosis of OS patients. Functional assays revealed that circ-ANKS1B silencing significantly decreased cell proliferation, migration and invasion abilities in vitro, and tumor formation ability in vivo. These findings indicated that circ-ANKS1B may act as a biomarker or therapeutic target for OS.

Recent studies have proved that circRNAs affected progression of tumor through specific sponging with miRNAs[6]. In present study, we demonstrated that miR-149-5p was target sponging by circ-ANKS1B through using bioinformatics analysis and luciferase reporter assay. Many studies have proved that miR149 could act as a tumor suppressor role during progression of solid tumor [25, 26]. Moreover, miR-149 was proved markedly downregulated in human OS tissues and could significantly inhibit proliferation and migration of OS cells[27]. To explore the deep molecular mechanism between circ-ANKS1B and miR-149$5 p$, functional experiments were conducted to investigate the interaction between them during progression of OS. Firstly, we found that expression of circ-ANKS1B and miR-149 was negative correlation both in human OS tissues and cell lines. Secondly, we also confirmed that circ-ANKS1B inhibited expression of miR-149-5p in OS cells. More importantly, the inhibitory effect of proliferation and invasion caused by miR-149-5p could be recovered by circ-ANKS1B overexpression, demonstrating that circ-ANKS1B exerted oncogene role in OS via negatively targeting miR-149-5p.

It is widely acknowledged that miRNAs could regulate gene expression post-transcriptionally[28]. Hence, we further investigated the downstream mechanism of miR-149-5p in OS cells. Through bioinformatics analysis and functional experiments, we identified Ki-67 as a potential target of miR-149-5p. Moreover, we also confirmed that circ-ANKS1B promoted Ki-67 expression via inhibiting miR-149-5p in OS cells. Ki-67 is commonly used for evaluating proliferate ability of cells because its abnormal expression is associated with cell growth[29]. High level of Ki67 had been proved to reflect the high cellular proliferation rate in most aggressive tumors, including OS[30]. To determine the effect of circ-ANKS1B in miR-149-5p / Ki-67 signaling axis, we preformed rescued experiments. We found both mRNA and protein levels of Ki-67 were decreased in OS cells under the condition of miR-149-5p mimics, while such phenomenon was restored by adding the circ-ANKS1B. Additionally, we testified the decreased Ki-67 expression in tumors from mice treated with circ-ANKS1B-delepted OS cells. All these results suggested that circ-ANKS1B positively regulated Ki-67 by interacting with miR-149-5p. Nevertheless, considering the specific configuration and ambiguous function, the more detailed molecular mechanism of circ-ANKS1B involved in the tumorigenesis of OS is still inconclusive and needs to be further studied.

\section{Conclusion}

In summary, the expression of circ-ANKS1B was upregulated in OS cell lines and tissues. Furthermore, its expression was associated with poor prognosis of OS patients. circ-ANKS1B silencing significantly suppressed OS cell proliferation and invasion in vitro and in vivo. Mechanistically, circ-ANKS1B could 
promote the expression of $\mathrm{Ki}-67$ by acting as a sponge of miR-149-5p. These results demonstrated that the circ-ANKS1B/miR-149-5p/Ki-67 axis may play critical regulatory roles in the pathogenesis of OS and may serve as a novel therapy target in OS.

\section{Declarations}

\section{Authors' contributions}

H.Y, Y.J.L and L.M.W conceived and designed the study. H.Y, C.L, Y.L.L, L.Z and Y.Q.W performed experiments; Y.Z, C.L and M.Z collected the clinical samples; All authors reviewed and approved the manuscript.

\section{Acknowledgments}

Not applicable

\section{Funding}

Not applicable

\section{Disclosure of Potential Conflict of Interest}

The authors report no conflicts of interest in this work.

\section{Availability of data and materials}

All the data and material could be traced from the paper we have published before

\section{Consent for publication}

All the listed authors have participated actively in the study, and have seen and approved the submitted manuscript.

\section{Ethics approval and consent to participate}

The study was approved by the human ethic committee of The First Affiliated Hospital of Zhengzhou University. All patients provided written informed consent and the project was in accordance with the Helsinki Declaration of 1975.

\section{Abbreviations}

Hepatocellular Carcinoma (HCC); overall survival (OS) \Tissue microarrays (TMA); Quantitative real-time PCR (qRT-PCR); Cell counting kit-8 (CCK-8); 3'UTR (3' untranslated region); The gene set enrichment analysis (GSEA)『standard deviation (SD); 


\section{References}

1. Ottaviani G, Jaffe N: The Epidemiology of Osteosarcoma. Cancer Treatment \& Research 2009, 152:3.

2. Luetke A, Meyers PA, Lewis I, Juergens H: Osteosarcoma treatment - where do we stand? A state of the art review. Cancer Treatment Reviews 2014, 40(4):523-532.

3. Anderson ME: Update on Survival in Osteosarcoma. Orthopedic Clinics of North America 2016, 47(1):283.

4. Memczak S, Jens M, Elefsinioti A, Torti F, Krueger J, Rybak A, Maier L, Mackowiak SD, Gregersen LH, Munschauer M: Circular RNAs are a large class of animal RNAs with regulatory potency. Nature 2013, 495(7441):333-338.

5. WR J, JA S, K W, MK S, CE B, J L, WF M, NE S: Circular RNAs are abundant, conserved, and associated with ALU repeats. RNA (New York, NY) 2013, 19(2):141-157.

6. LS K, MS A, LVW S, KK E, TB H, J K: The biogenesis, biology and characterization of circular RNAs. Nature reviews Genetics 2019, undefined(undefined):undefined.

7. X Z, Y C, J X: Circular RNAs: Biogenesis, Mechanism, and Function in Human Cancers. International journal of molecular sciences 2019, 20(16):undefined.

8. Wang D, Yang S, Wang H, Wang J, Zhang Q, Zhou S, He Y, Zhang H, Deng F, Xu H: The progress of circular RNAs in various tumors. Am J Transl Res 2018, 10(6):1571-1582.

9. HB M, YN Y, JJ Y, XX C, HF L: Extensive profiling of circular RNAs and the potential regulatory role of circRNA-000284 in cell proliferation and invasion of cervical cancer via sponging miR-506. American journal of translational research 2018, 10(2):592-604.

10. H L, X J, B L, P Z, W C, Q L: CircRNA CBL.11 suppresses cell proliferation by sponging miR-6778-5p in colorectal cancer. BMC cancer 2019, 19(1):826.

11. X Z, D N, Z Q, D W, Z T, X C, B W, X H: Identification and functional characterization of circRNA0008717 as an oncogene in osteosarcoma through sponging miR-203. Oncotarget 2018, 9(32):22288-22300.

12. $H Z, G$ W, C D, P L, R W, W D, D T, D W, C L, Q W et al: Increased circular RNA UBAP2 acts as a sponge of miR-143 to promote osteosarcoma progression. Oncotarget 2017, 8(37):61687-61697.

13. Z W, W S, C J: Overexpressing circular RNA hsa_circ_0002052 impairs osteosarcoma progression via inhibiting Wnt/ $\beta$-catenin pathway by regulating miR-1205/APC2 axis. Biochemical and biophysical research communications 2018, 502(4):465-471.

14. L H, M C, J P, W Y: Circular RNA circNASP modulates the malignant behaviors in osteosarcoma via miR-1253/FOXF1 pathway. Biochemical and biophysical research communications 2018, 500(2):511-517.

15. J J, A C, W Q, Y C, Q L, X Z, D J: Dysregulated circRNA_100876 suppresses proliferation of osteosarcoma cancer cells by targeting microRNA-136. Journal of cellular biochemistry 2019, 120(9):15678-15687. 
16. $C L, Y C, P B, J W, Z L, T W, Q$ C: LDHB may be a significant predictor of poor prognosis in osteosarcoma. American journal of translational research 2016, 8(11):4831-4843.

17. $Q H, C L, Y C, J B, K L, R S, X C, J L, X W$ : CBX2 is a functional target of miRNA let-7a and acts as a tumor promoter in osteosarcoma. Cancer medicine 2019, 8(8):3981-3991.

18. C X, Y H, W Z, X C, Y Y, Q H, J C, L L, F R, Z R et al: Low expression of LACTB promotes tumor progression and predicts poor prognosis in hepatocellular carcinoma. American journal of translational research 2018, 10(12):4152-4162.

19. Chen J, Y Y, X C, Y H, Q H, H L, Q H, FR, J L, C L: MiR-139-5p is associated with poor prognosis and regulates glycolysis by repressing PKM2 in gallbladder carcinoma. Cell Proliferation 2018, 51(11):e12510.

20. J C, Y Y, H L, Q H, X C, Y H, C X, F R, Z R, J L et al: Long non-coding RNA PVT1 promotes tumor progression by regulating the miR-143/HK2 axis in gallbladder cancer. Molecular cancer 2019, 18(1):33.

21. J B, Y Y, J C, Y H, X C, Z R, C X, L L, Q H, J L et al: MiR-126 negatively regulates PLK-4 to impact the development of hepatocellular carcinoma via ATR/CHEK1 pathway. Cell death \& disease 2018, 9(10):1045.

22. S H, X Z, B G, P S, CT H, J P, S T, J Y: A novel circular RNA hsa_circ_0008035 contributes to gastric cancer tumorigenesis through targeting the miR-375/YBX1 axis. American journal of translational research 2019, 11(4):2455-2462.

23. Y J, T W, W L, R Z, M C: Circular RNA hsa_circ_0002024 suppresses cell proliferation, migration, and invasion in bladder cancer by sponging miR-197-3p. American journal of translational research 2019, 11(3):1644-1652.

24. H C, B H, L J, X R, Z Z: Hsa_circ_0103809 promotes cell proliferation and inhibits apoptosis in hepatocellular carcinoma by targeting miR-490-5p/SOX2 signaling pathway. American journal of translational research 2018, 10(6):1690-1702.

25. L L, Y C, Q L, P D: IncRNA HNF1A-AS1 modulates non-small cell lung cancer progression by targeting miR-149-5p/Cdk6. Journal of cellular biochemistry 2019, undefined(undefined):undefined.

26. $D L, R Y, L Y, D$ W, X Z, Y S: circANKS1B regulates FOXM1 expression and promotes cell migration and invasion by functioning as a sponge of the miR-149 in colorectal cancer. OncoTargets and therapy 2019, 12(undefined):4065-4073.

27. RD X, F F, XS Y, ZD L, LF L: miR-149-5p inhibits cell growth by regulating TWEAK/Fn14/PI3K/AKT pathway and predicts favorable survival in human osteosarcoma. International journal of immunopathology and pharmacology 2018, 32(undefined):2058738418786656.

28. Macfarlane LA, Murphy PR: MicroRNA: Biogenesis, Function and Role in Cancer. Current Genomics 2010, 11(7):-

29. P V, S C, V M, B D, H M: Ki67 index and S-phase fraction in human breast carcinomas. Comparison and correlations with prognostic factors. American journal of clinical pathology 1990, 94(6):681-686. 
30. Mardanpour K, Rahbar M, Mardanpour S: Coexistence of her2, ki67, and p53 in osteosarcoma: A strong prognostic factor. North American Journal of Medical Sciences 2016, 8(5):210-214.

\section{Tables}

Table 1. Correlation of clinic-pathological features with circ-ANKS1B expression in osteosarcoma TMA corhort

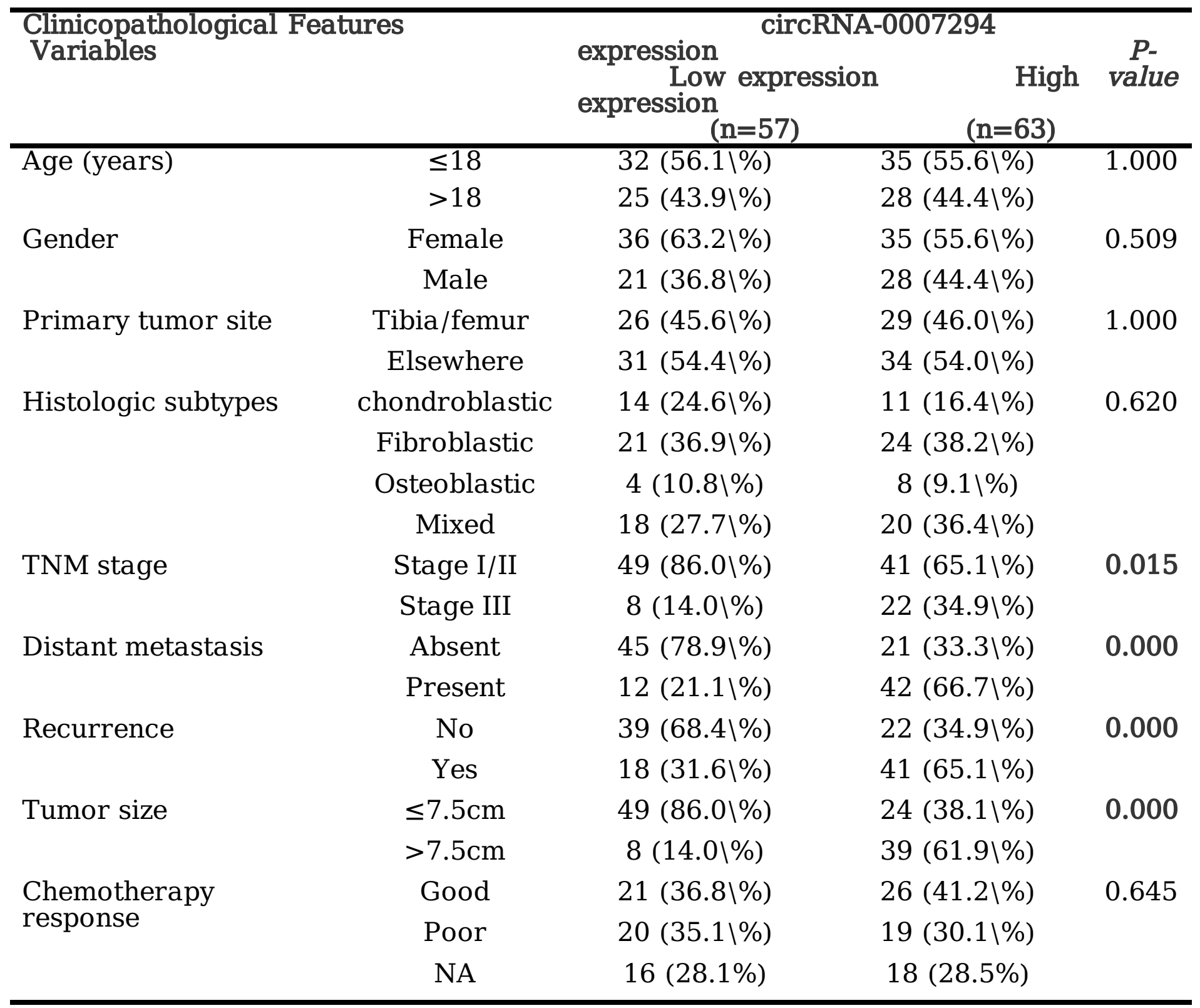

a Bold values indicate statistical significance, $\mathrm{P}<0.05$

Table 2. Correlation of clinic-pathological features with circ-ANKS1B expression in OS cohort 


Univariate
analysis
HR $95 \% \quad P$ value
CI $\quad \begin{gathered}\text { Multivariate } \\ \text { analysis } \\ 95 \% \quad P \text { value } \\ \end{gathered}$

Univariate and multivariate analysis of overall survival in OS patients $(\mathrm{n}=120)$

\begin{tabular}{llll}
\hline Age ( $>18$ vs $\leq 18)$ & 1.167 & $0.906-$ & 0.188 \\
& & 1.463 &
\end{tabular}

Gender (Male vs Female) $\quad 1.017 \quad 0.882-\quad 0.256$

Primary tumor site $0.905 \quad 0.721-0.376$

(Tibia/femur $\quad$ vs $\quad 1.081$

Elsewhere)

TNM stage (III/IV vs I/II) $3.584 \quad 2.473-\quad 0.001 \quad 3.097 \quad 2.469-\quad 0.003$

$\begin{array}{llllllll}\text { Distant metastasis } & 3.081 & 2.989- & 0.005 & 2.601 & 2.022- & 0.034\end{array}$

(Present vs Absent) $\quad 4.326$

Recurrence (Present vs 3.050 2.303-

Absent) 3.377

Tumor size $(>7.5 \mathrm{~cm}$ vs $1.604 \quad 1.279$ $\leq 7.5 \mathrm{~cm})$

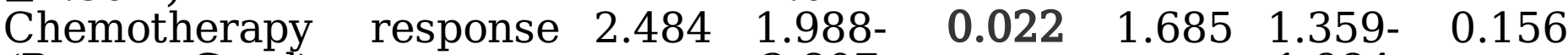
(Poor vs Good) 2.807 circ-ANKS1B expression $2.590 \quad 1.989$ (High vs Low) 3.067
$0.0112 .598 \quad 1.978$
0.026

3.341

Univariate and multivariate analysis of disease-free survival in OS patients $(\mathrm{n}=120)$

\begin{tabular}{llll}
\hline Age $(>18$ vs $\leq 18)$ & 1.047 & $0.783-$ & 0.315 \\
& & 1.247 &
\end{tabular}

Gender (Male vs Female) $\quad 0.970 \quad 0.878-0.375$

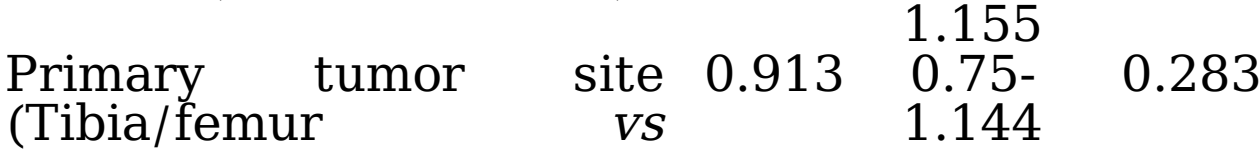

Elsewhere)

TNM stage (III/IV vs I/II) $3.256 \quad 2.479$

Distant 3.651

Distant metastasis $2.732 \quad 2.008$

(Present vs Absent)

3.196

Recurrence (Present vs 2.219 2.058-

Absent)

2.717

Tumor size $(>7.5 \mathrm{~cm}$ vs 1.580 1.385$\leq 7.5 \mathrm{~cm}$ )

1.895

Chemotherapy response $2.127 \quad 1.904$ -

(Poor vs Good)

2.555

circ-ANKS1B expression 2.365

2.030

(High vs Low)

3.206

0.023

2.987

$2.278-$

3.755

$0.019 \quad 3.019 \quad 2.330$

3.606

$0.016 \quad 2.135 \quad 1.955$

3.347

0.005

0.031

0.027

0.089

$\begin{array}{llll}0.040 & 1.795 & 1.438- & 0.195\end{array}$

2.214

$\begin{array}{llll}0.026 & 2.426 & 2.124- & 0.019\end{array}$

\section{Figures}


A

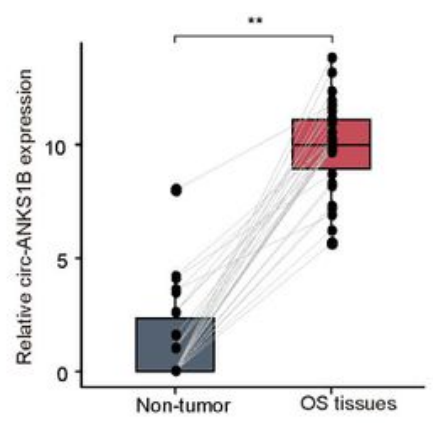

B circ-ANKS1B expression $\begin{array}{lllll}-1.0 & -0.5 & 0.0 & 0.5 & 1.0\end{array}$

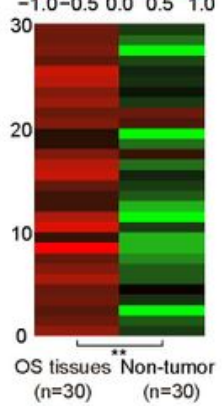

C

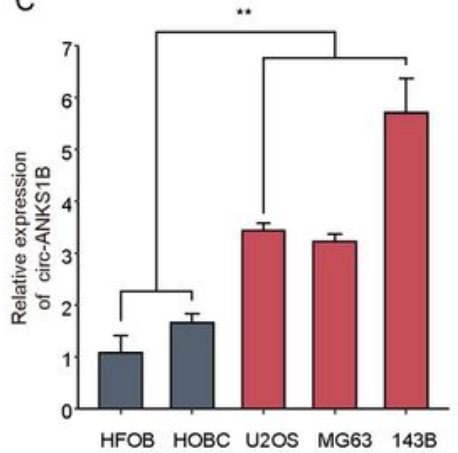

E

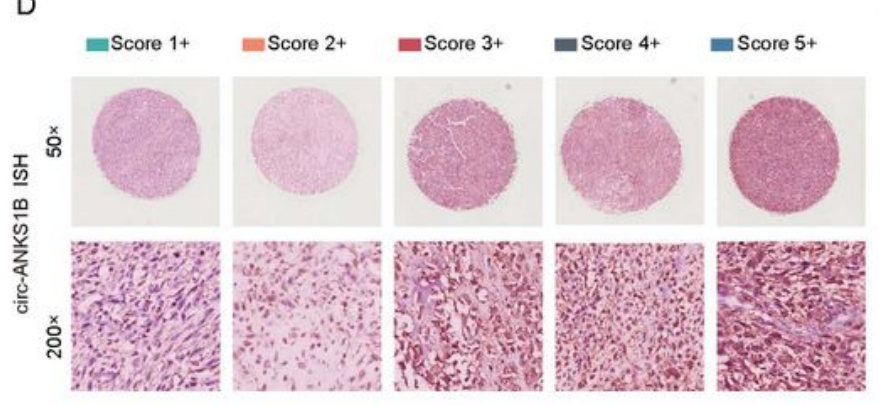

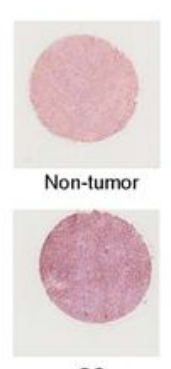

os

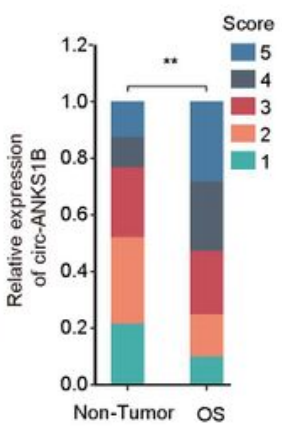

\section{Figure 1}

circ-ANKS1B expression is upregulated in OS tissues and cell lines (A-B) Relative expression of circANKS1B in human OS tissues $(n=30)$ compared with corresponding adjacent normal tissues $(n=30)$. circANKS1B expression was examined by qPCR and normalized to GAPDH expression. (C) circ-ANKS1B expression levels of OS cell lines (U2OS, MG63 and 143B), compared with that in human osteoblast cells (HFOB and HOBC). (D) Representative circ-ANKS1B staining patterns via ISH assays. (E) circ-ANKS1B 
expression was significantly higher in OS tissues $(n=120)$ compared with that in non-tumor tissues $(n=$ 65). ${ }^{*} p<0.05,{ }^{* \star} p<0.01$.

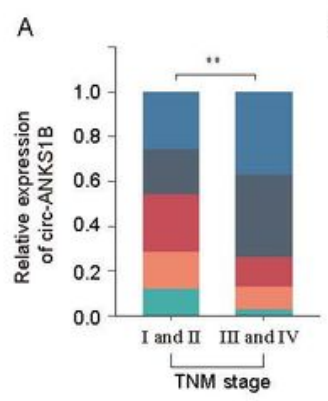

E
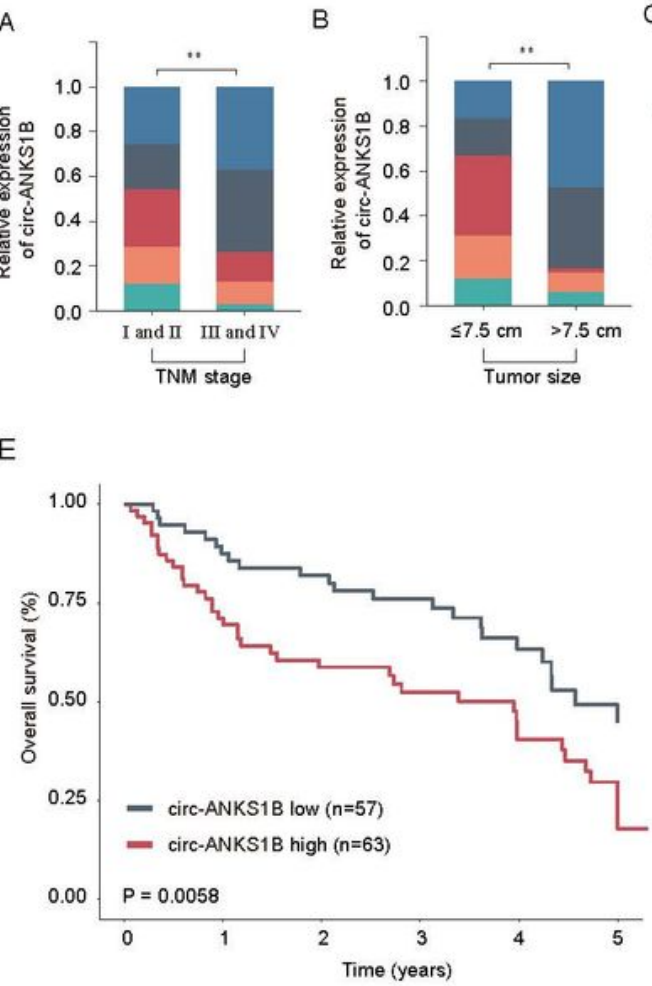

C
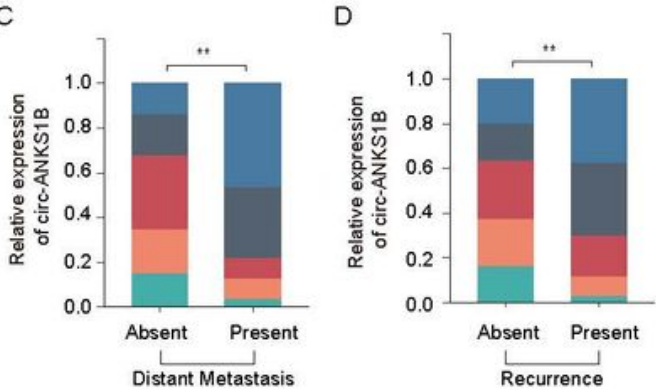

F

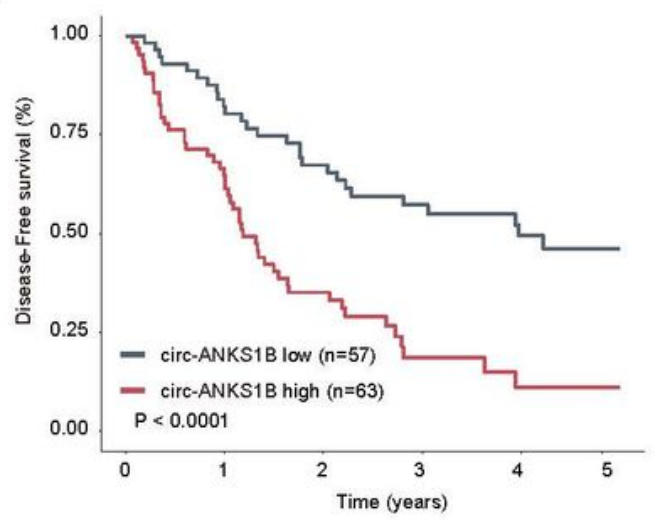

Figure 2

circ-ANKS1B expression predicts OS patients' survival. The correlation of circ-ANKS1B expression level with TNM stage (A), tumor size (B), distant metastasis (C) and recurrence status (D). Kaplan-Meier 
analysis revealed that high expression of circ-ANKS1B was related to poorer overall survival (E) and disease-free survival (F) of OS patients. ${ }^{*} p<0.05,{ }^{*} p<0.01$.
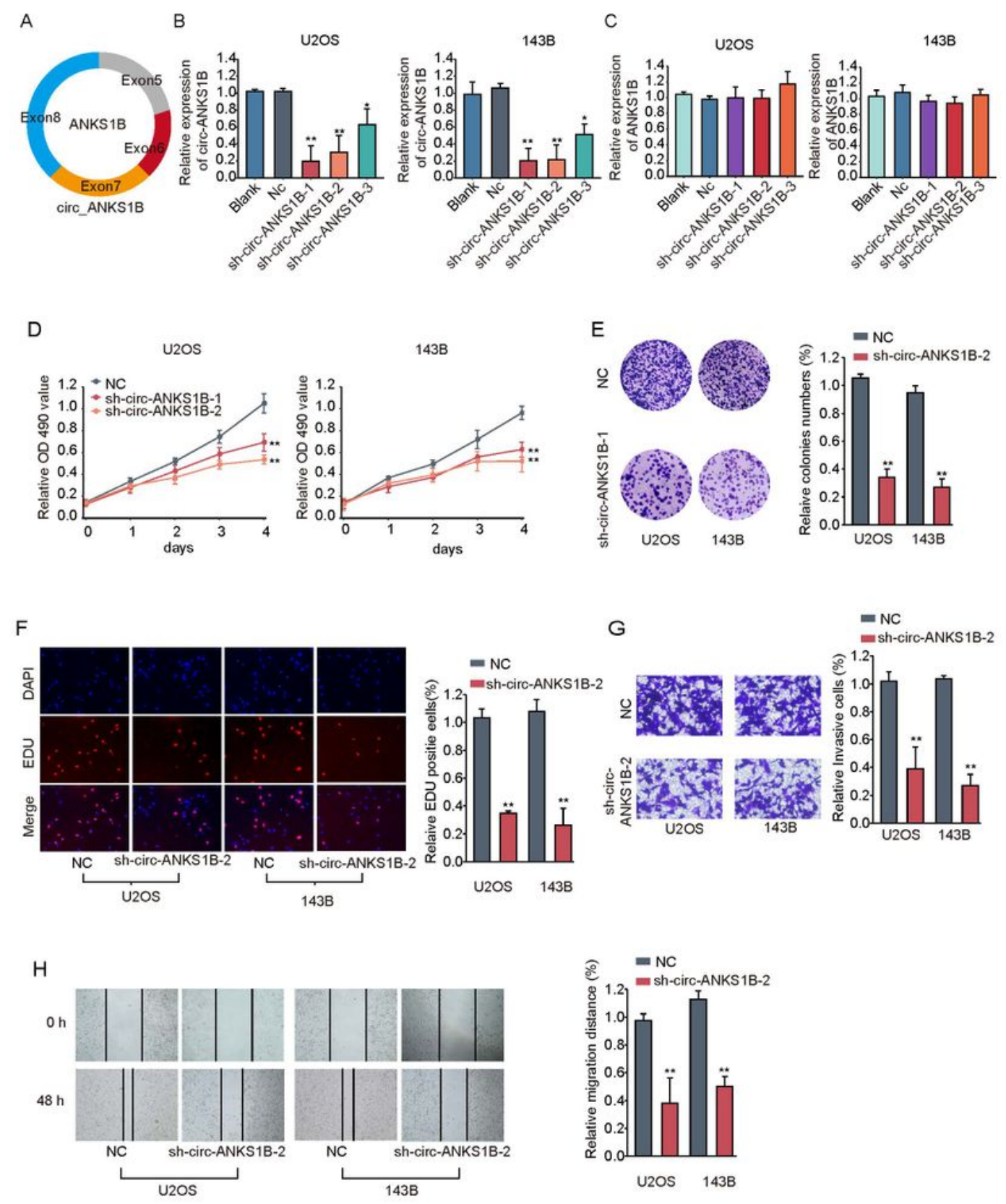

\section{Figure 3}

Silencing of circ-ANKS1B inhibits OS cell proliferation, migration and invasion in vitro. (A-C) Expression levels of circ-ANKS1B and its linear mRNA ANKS1B were detected by qRT-PCR in U2OS and 143B cells transfected with shRNAs targeting circ-ANKS1B (sh-circ-ANKS1B-1/2/3) or negative control 
oligonucleotide (NC). (D) CCK-8 assay showed that the proliferation of U2OS and 143B cells was inhibited with circ-ANKS1B silencing. (E) Colony formation assays suggested that circ-ANKS1B knockdown inhibited the colony-forming ability of U2OS and 143B cells. (F) Edu assay showed that the DNA synthetics of U2OS and 143B cells was inhibited with circ-ANKS1B silencing. (G, H) Transwell and wound healing assays showed that circ-ANKS1B knockdown significantly inhibited the migration and invasion of U2OS and 143B cells. Data are listed as means \pm s.d. of at least three independent experiments. ${ }^{*} \mathrm{p}<0.05,{ }^{\star *} \mathrm{p}<0.01$.

A

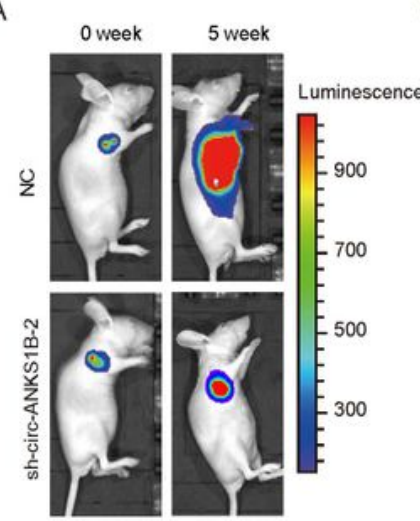

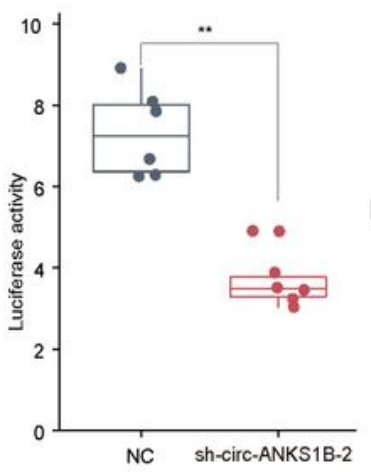

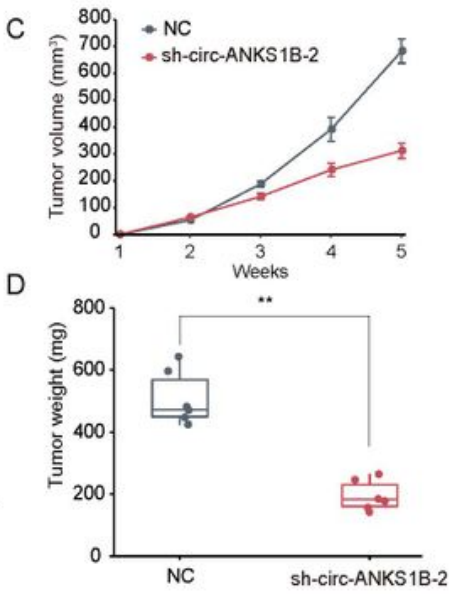

E
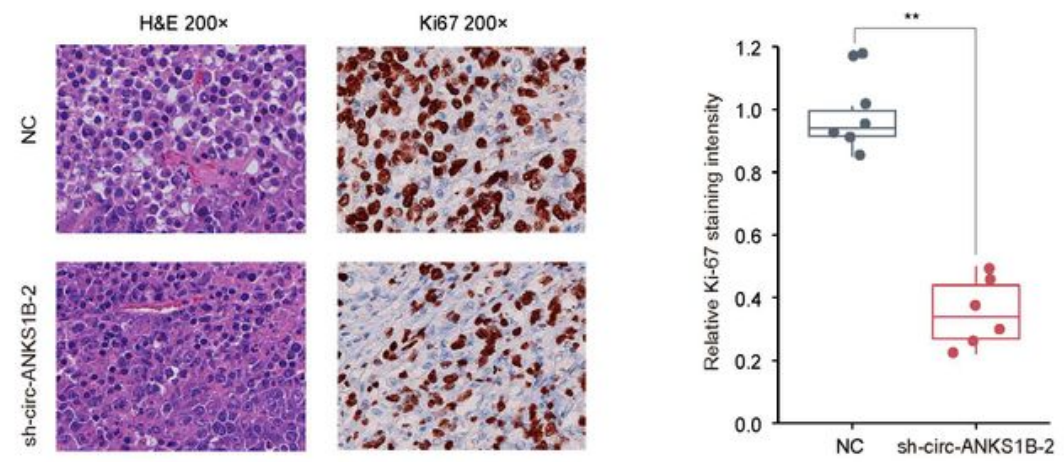


\section{Figure 4}

Knockdown of circ-ANKS1B suppresses OS tumorigenesis in vivo. U2OS cells stably transfected with shcirc-ANKS1B (sh-circ-ANKS1B-2) or negative control (NC) were implanted subcutaneously into nude mice and tumor growth was monitored. (A) Representative living image photos of nude mice. (B) The relative photon flux of tumors from NC or sh-circ-ANKS1B-2 group was analyzed. $n=6$ per group. (C, D) The relative tumor volume and tumor weight of tumors from NC or sh-circ-ANKS1B group was analyzed. $n=6$ per group. (E) Representative IHC staining and the expression levels of Ki-67 in tumor tissues from NC or sh-circ-ANKS1B-2 group. ${ }^{*} p<0.05,{ }^{* *} p<0.01$. 
Wt circ-ANKS1B 5' CUUGGGAAAAAUGGAAGCCAGAG 3'

I I | | I I

hsa-miR-149-5p 3' C CCUCACUUCUGUGCCUCGGUCU

Mut circ-ANKS1B 5' ACACCAGGCCUCACGCAUUUACC 3'
B

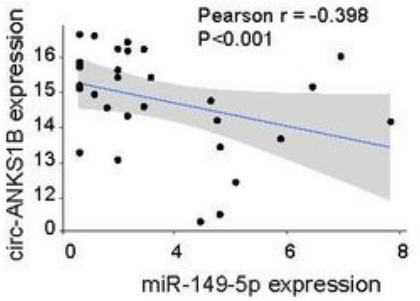

C

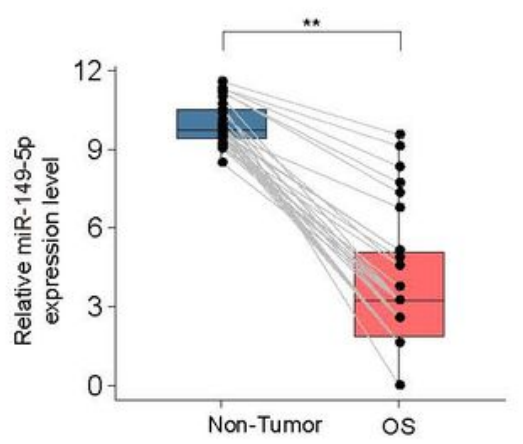

E

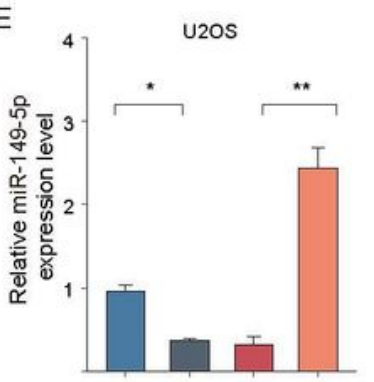

- NC

- pcDNA3.1-circ-ANKS1B

miR-149-5p mock

\&pCDNA3.1-circ-ANKS1B

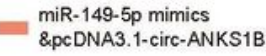

D

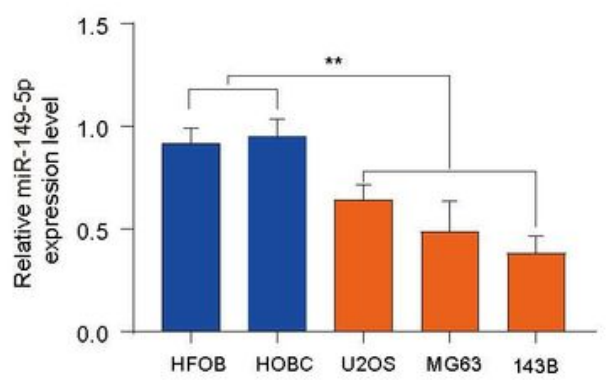

F 8] * U2OS

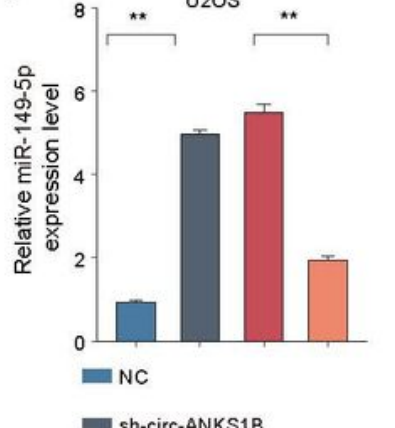

G

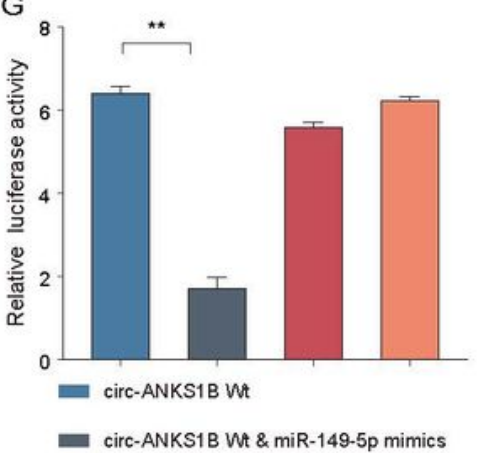

miR-149-5p mock \& sh-circ-ANKS1B

circ-ANKS1B Mut

miR-149-5p ihibitor \& sh-circ-ANKS1B $=$ circ-ANKS1B Mut \& miR-149-5p mimics

\section{Figure 5}

The interaction between circ-ANKS1B and miR-149-5p. (A) The putative binding sites of miR-149-5p on circ-ANKS1B are predicted. (B) The correlation between miR-149-5p and circ-ANKS1B expression in OS tissues. (C) RT-qPCR analysis of miR-149-5p expression levels in OS tissues $(n=30)$ compared with corresponding adjacent normal tissues ( $n=30)$. (D) miR-149-5p expression levels of OS cell lines (U2OS, MG63 and 143B), compared with that in human osteoblast cells (HFOB and HOBC). RT-qPCR analysis of 
miR-149-5p expression levels in U2OS and 143B cells after circ-ANKS1B overexpression (E) or silencing (F). (G) Dual-luciferase reporter assay showed the luciferase activity of the combination between miR149-5p and circ-ANKS1B. * $p<0.05,{ }^{* *} p<0.01$.
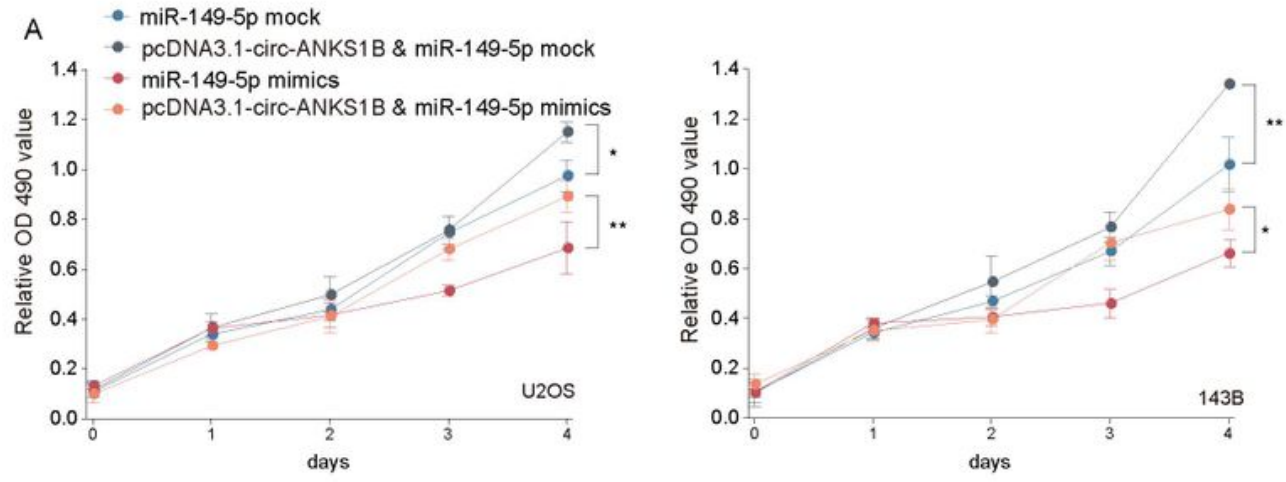

B

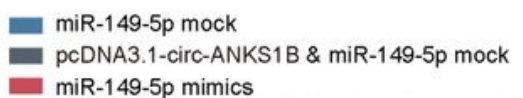

$$
\text { miR-149-5p mimics }
$$

pCDNA3.1-circ-ANKS1B \& miR-149-5p mimics

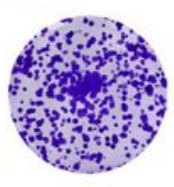

(

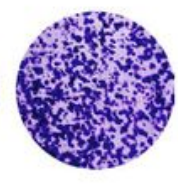

-
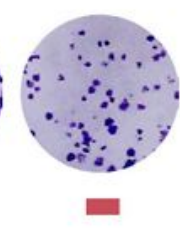

-

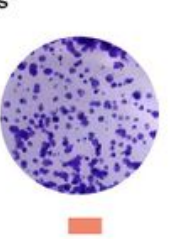

近

c

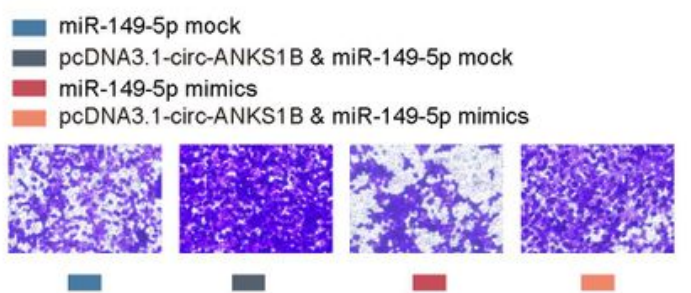

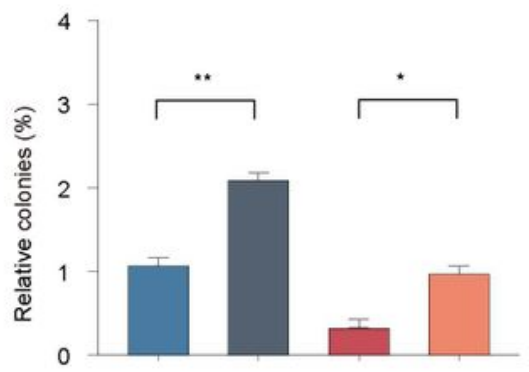

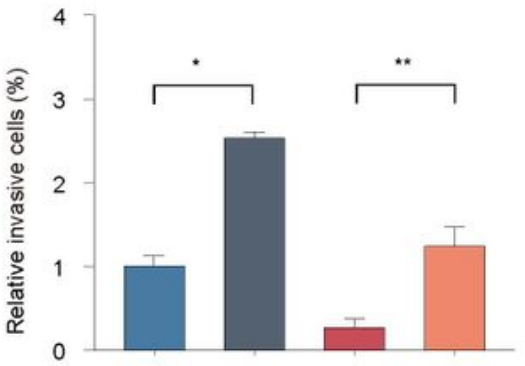

\section{Figure 6}

Overexpression of circ-ANKS1B partially reverses the suppressive effect of miR-149-5p in OS cells. U2OS and 143B cells were transfected with miR-149-5p mock or miR-149-5p mimics, with or without circ- 
ANKS1B overexpression vector. (A) Cell proliferation of U2OS and 143B was analyzed at indicated time points by CCK-8 kit. Colony formation (B) and cell invasion ability (C) of U2OS was analyzed by colony formation assay and transwell assay, respectively. ${ }^{\star} p<0.05,{ }^{* *} p<0.01$.

A

Wt Ki-67 5' CCCAGUGUCUGGCGGGGAGCCAGG 3'

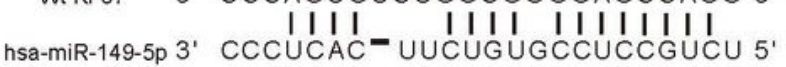

Mut Ki-67 5' CAUgaAcCGUCUgUaAuCgCGUa 3'

C

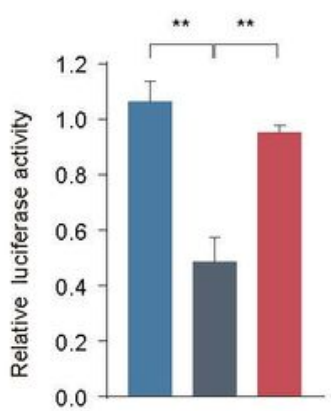

WT Ki-67 \& mimics NC

WT Ki-67 \& miR-149-5p mimics

Mut Ki-67 \& miR-149-5p mimics
$\mathrm{D}=$ Blank

$$
\begin{aligned}
& \text { = mimics NC } \\
& \text { - inhibitor NC mimics }
\end{aligned}
$$$$
\text { - miR-149-5p inhibitor }
$$

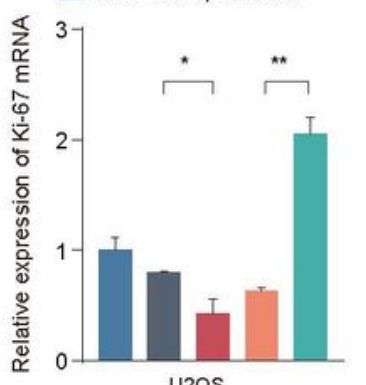

U2OS zzu cohort

B
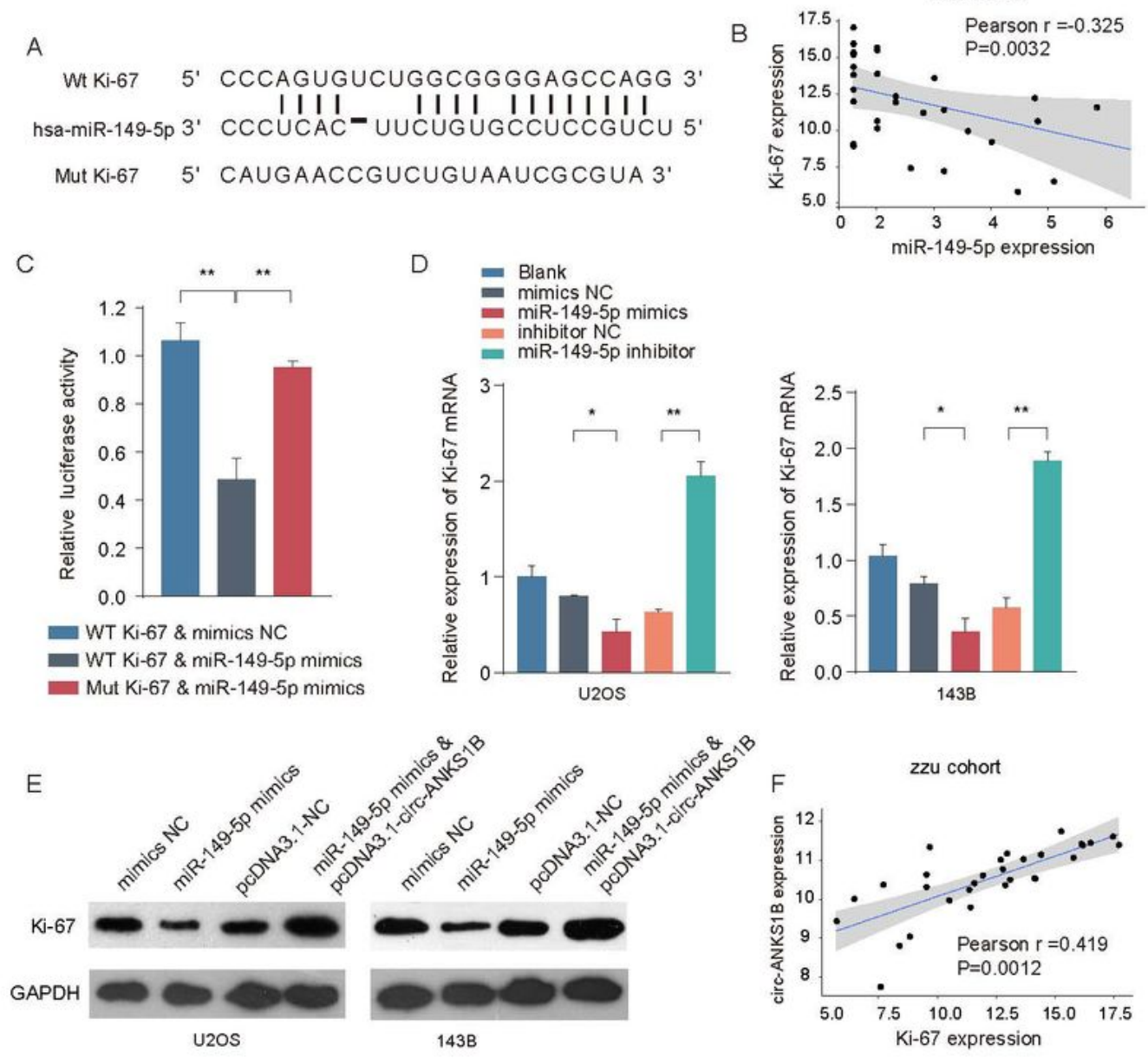

\section{Figure 7}

circ-ANKS1B inhibits Ki-67 expression by regulating miR-149-5p (A) The putative binding sites of miR149-5p on Ki-67 are predicted. (B) Correlation of the Ki-67 mRNA expression level between miR-149-5p 
expression. (C) Dual-luciferase reporter assay showed the luciferase activity of the combination between miR-149-5p and Ki-67. The mRNA expression level (D) and protein level (E) of Ki-67 expression was significantly decreased by miR-149-5p mimics, but this effect could be restored by circ-ANKS1B overexpression. (F) Correlation of the Ki-67 mRNA expression level and circ-ANKS1B expression. ${ }^{*} \mathrm{p}<$ $0.05, * * p<0.01$.
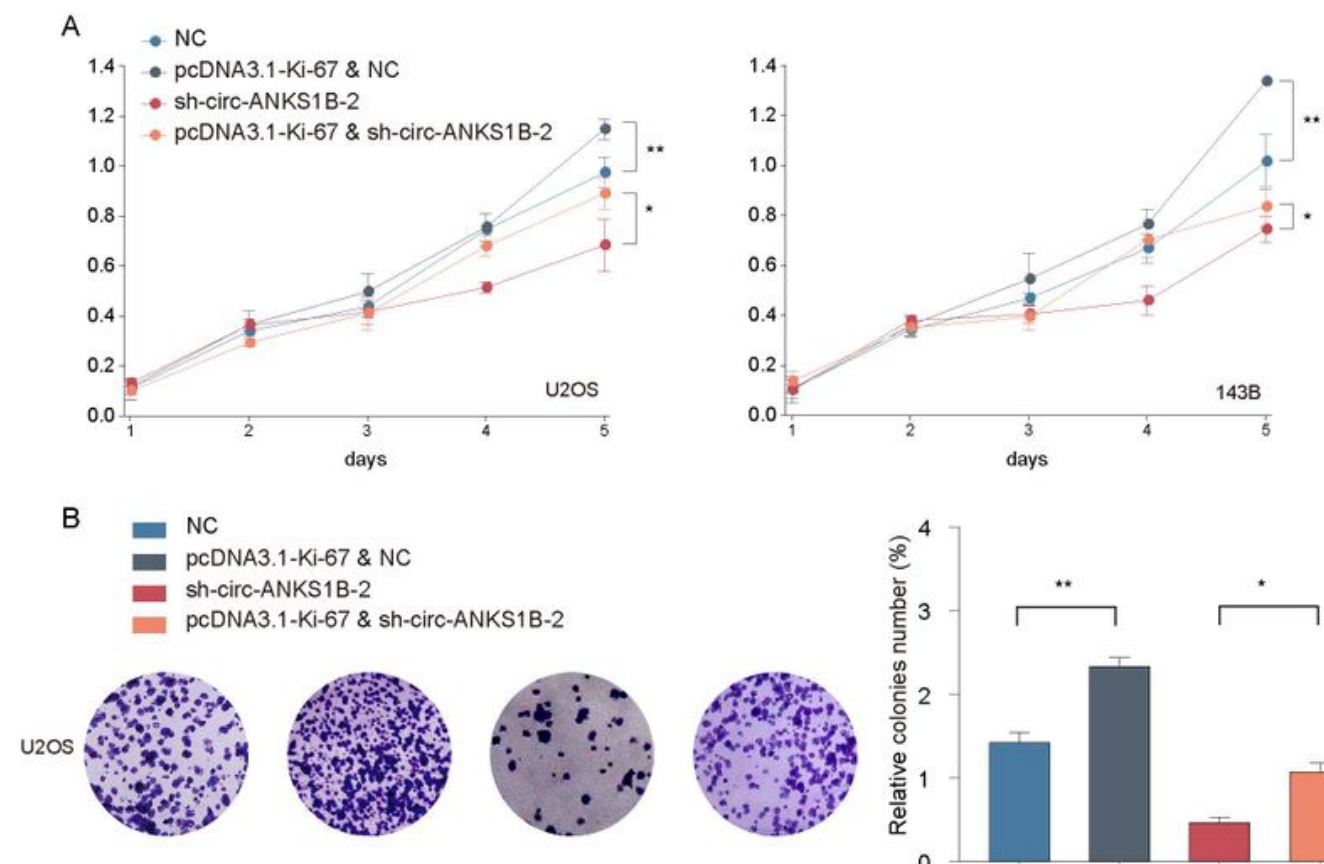

pCDNA3.1-Ki-67 \& NC

sh-circ-ANKS1B-2

pcDNA3.1-Ki-67 \& sh-circ-ANKS1B-2
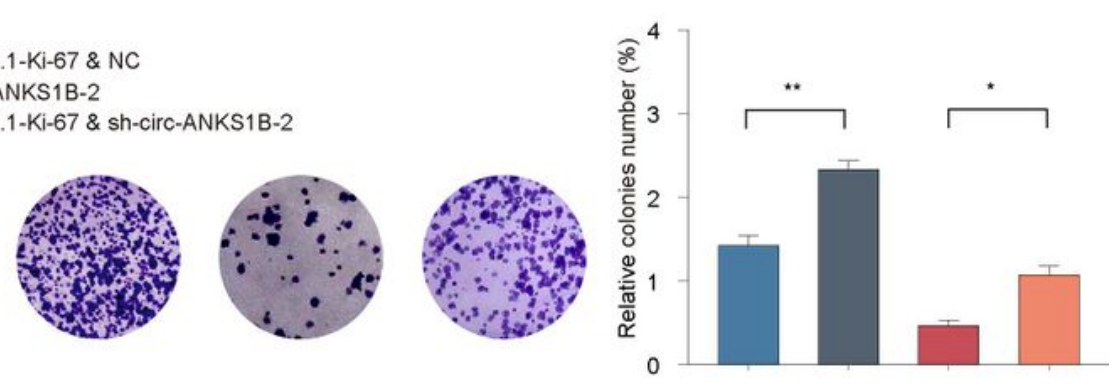

C
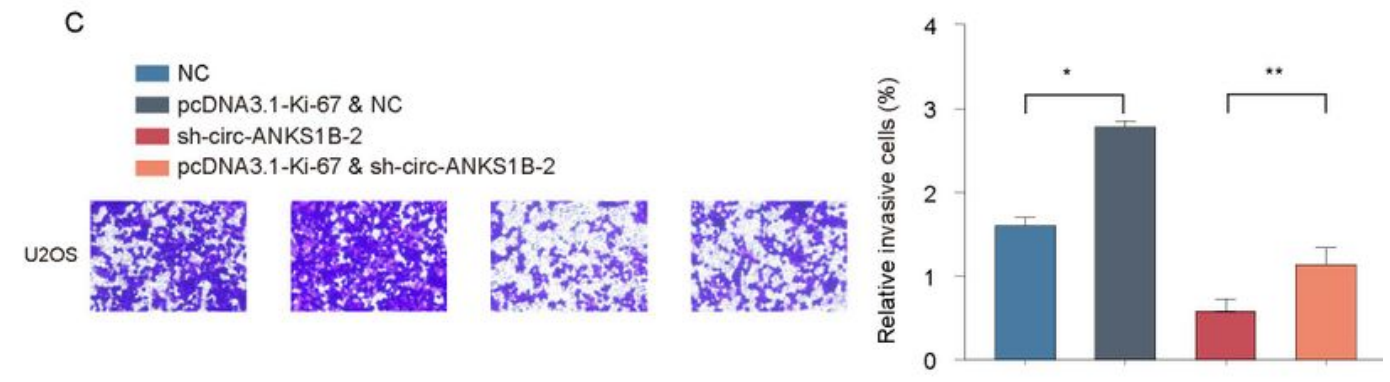

Figure 8 
circ-ANKS1B promote OS cell proliferation and invasion through upregulating Ki-67. U2OS and 143B cells were transfected with sh-circ-ANKS1B-2 or NC, with or without pcDNA3.1 overexpressed Ki-67. (A) Cell proliferation of U2OS and 143B was analyzed at indicated time points by CCK-8 kit. (B) Colony formation and cell invasion ability (C) of U2OS was analyzed by colony formation assay and transwell assay, respectively. ${ }^{*} p<0.05, * \star p<0.01$.

\section{Supplementary Files}

This is a list of supplementary files associated with this preprint. Click to download.

- supplementaryTable12.docx 